\title{
Nonlinear Forecasting Measurement of Magnetoencephalogram Recordings from Alzheimer's Disease Patients
}

\author{
Carlos Gómez*, Student Member, IEEE, Roberto Hornero, Member, IEEE, Ángela Mediavilla, \\ Alberto Fernández, and Daniel Abásolo, Member, IEEE
}

\begin{abstract}
The goal of this study was to analyze the magnetoencephalogram (MEG) background activity in patients with Alzheimer's disease (AD) using a nonlinear forecasting measure. It is a nonparametric method to quantify the predictability of time series. Five minutes of recording were acquired with a 148-channel whole-head magnetometer in 15 patients with probable $A D$ and 15 elderly control subjects. Stationary epochs of 5 seconds $(848$ points, sample frequency of $169.55 \mathrm{~Hz}$ ) were selected. Our results showed that AD patients' MEGs were more predictable than controls' recordings. Additionally, an accuracy of $76.7 \%(80.0 \%$ sensitivity; $73.3 \%$ specificity) was reached using a receiver operating characteristic curve. These preliminary results suggest the usefulness of nonlinear forecasting to gain a better understanding of dynamical processes underlying the MEG recording.
\end{abstract}

\section{INTRODUCTION}

A LZHEIMER'S DISEASE (AD) is a primary degenerative neurological disorder of unknown etiology that gradually destroys brain cells. It is considered the main cause of dementia in western countries [1]. AD is characterized by neuronal loss and the appearance of neuritic plaques containing amyloid- $\beta$-peptide and neurofibrillary tangles. Clinically, this disease manifests as a slowly progressive impairment of mental functions whose course lasts several years prior to death [2]. Usually, AD starts by destroying neurons in parts of the patient's brain that are responsible for storing and retrieving information. Then, it affects the brain areas involved in language and reasoning. Eventually, many other brain regions are atrophied. Thus, $\mathrm{AD}$ patients may wander, be unable to engage in conversation, appear non-responsive, become helpless and need complete care and attention [3]. Although a definite $\mathrm{AD}$ diagnosis is only possible by necropsy, a differential diagnosis with other types of dementia and with major depression should be attempted. The differential diagnosis includes medical history studies, physical and neurological evaluation, mental status tests, and neuroimaging techniques.

Manuscript received April 7, 2008. This work was supported in part by Consejería de Educación de la Junta de Castilla y León under projects VA108A06 and VA102A06. Asterisk indicates corresponding author.

C. Gómez, R. Hornero, A. Mediavilla, and D. Abásolo are with the Biomedical Engineering Group at Department of Signal Theory and Communications, E.T.S. Ingenieros de Telecomunicación, University of Valladolid, Campus Miguel Delibes, 47011 - Valladolid, Spain (e-mail: cargom@tel.uva.es).

A. Fernández is with the Centro de Magnetoencefalografía Dr. PérezModrego, University Complutense of Madrid, Spain.
Nowadays, magnetoencephalography (MEG) and electroencephalography (EEG) recordings are not used in AD clinical diagnosis. Nevertheless, several studies have demonstrated that the analysis of EEG/MEG recordings could help physicians in the $\mathrm{AD}$ diagnosis (for review, see [2] and [4]). EEG and MEG are the only signals that record the synchronous oscillations of cortex pyramidal neurons directly and non-invasively. MEG is a measure of the magnetic brain activity, whereas EEG records the electrical activity of the brain. However, the use of MEG recordings to study the brain background activity offers significant advantages over EEG. The magnetic fields are less distorted by the resistive properties of the skull [5]. Additionally, MEG provides reference-free recordings. Furthermore, the spatial resolution is higher in MEG than in conventional EEG [5]. On the other hand, the magnetic signals generated by the human brain are extremely weak. SQUID (Superconducting QUantum Interference Device) sensors and magnetically shielded rooms are necessary to detect them.

The electromagnetic brain activity (EEG and MEG) has been researched in the last decades by means of non-linear techniques. The most used to study the brain recordings from $\mathrm{AD}$ patients was the correlation dimension $[6,7]$. However, the theoretical limitations of this classical measure make necessary to study the MEG background activity with other non-linear techniques. In fact, Lempel-Ziv complexity $[8,9]$, approximate entropy [9], synchronization likelihood [10], and auto-mutual information [11] have been already used to analyze the MEG activity in AD.

In this preliminary study, we have examined the MEG background activity in patients with probable $\mathrm{AD}$ and in age-matched control subjects using a nonlinear forecasting $(N L F)$ measure. Our purpose is to test the hypothesis that the neuronal dysfunction in $\mathrm{AD}$ is associated with differences in the dynamical processes underlying the MEG recording.

\section{MATERIALS AND METHODS}

\section{A. MEG recording}

MEGs were recorded using a 148-channel whole-head magnetometer (MAGNES $2500 \mathrm{WH}, 4 \mathrm{D}$ Neuroimaging) located in a magnetically shielded room. The subjects lay comfortably on a patient bed, in a relaxed state and with their eyes closed. They were asked to stay awake and to avoid eye and head movements. For each subject, five minutes of recording were acquired at a sampling frequency 
of $678.17 \mathrm{~Hz}$. These recordings were down-sampled by a factor of four, obtaining a sampling rate of $169.55 \mathrm{~Hz}$. Data were digitally filtered between 0.5 and $40 \mathrm{~Hz}$. Finally, artifact-free epochs of 5 seconds (848 samples) were selected and copied as ASCII files to a personal computer for off-line analysis.

\section{B. Subjects}

MEG data were acquired from 30 subjects: 15 patients with probable $\mathrm{AD}$ and 15 elderly control subjects. Cognitive status was screened in both groups with the Mini Mental State Examination (MMSE). The AD group consisted of fifteen patients ( 5 men and 10 women; age $=72.33 \pm 9.04$ years, mean \pm standard deviation, SD) fulfilling the criteria of probable AD, according to the criteria of the National Institute of Neurological and Communicative Disorders and Stroke - Alzheimer's and Related Disorders Association (NINCDS-ADRDA). The mean MMSE score for the patients was $17.67 \pm 3.94$ points. Patients were free of other significant medical, neurological and psychiatric diseases than AD. Moreover, any of the participants in the study used medication that could be expected to influence in the MEG recording.

MEGs were also obtained from fifteen age-matched control subjects without past or present neurological disorders $(7$ men and 8 women; age $=72.53 \pm 5.40$ years, MMSE score $=29.00 \pm 0.33$ points). The local ethics committee approved this study. All control subjects and all caregivers of the patients gave their informed consent for the participation in the current study.

\section{Stationarity}

To estimate the NLF appropriately, stationary MEG epochs are necessary. We have employed the Bendat and Piersol's runs test, a general non-parametric test for weak or wide sense stationarity [12]. The assessment of stationarity with this method depends on the window size, which can be determined by roughly estimating the dominant low frequency band in terms of energy distribution [13]. We found that over half of the MEG epochs retained the majority of their energy below $10.6 \mathrm{~Hz}$. Thus, a window of at least $94.3 \mathrm{~ms}$ is necessary. As a conservative estimate, the windows size should be more than three times the previous value [13]. Thus, in our study, a window length of $300 \mathrm{~ms}$, that is 50 samples, was employed to test the stationarity. The stationary epochs of 848 samples were selected for further analysis with $N L F$ whereas the remainders were discarded.

\section{Nonlinear forecasting (NLF)}

Nonlinear forecasting $(N L F)$ was proposed in 1990 by Sugihara and May to measure the predictability of time series [14]. It is a nonparametric method, which uses no prior information about the model used to generate the time series [14]. Applied to a brain recordings, NLF may quantify the loss of memory of past brain states [15]. This measure has been used to study the behaviour of brain signals during different psychophysiological experiments [16]. Aftanas et al. [15] tested the EEG predictability during evoked emotions, finding that negative evoked emotions showed better predictability than neutral or positive ones. Additionally, EEG recordings from subjects with depression disorders [18] and with Creutzfeldt-Jakob disease [17] have been analyzed with $N L F$. The $N L F$ algorithm is as follows $[14,15]$ :

1) The data set (with size $n$ ) is divided into two parts of equal size. The first one $\left(y_{1}, y_{2}, \ldots, y_{n / 2}\right)$ is used as a learning set to build a model of the system dynamic, and the second one $\left(x_{1}, x_{2}, \ldots, x_{n / 2}\right)$ is a test set which is compared to the predicted series [16].

2) For an embedding dimension $m$ and a time delay $\tau$, the attractor of each signal is reconstructed using the Takens' embedding theorem [19]:

$$
\begin{gathered}
X_{t}=\left\{x_{t}, x_{t+\tau}, x_{t+2 \tau}, \ldots, x_{t+(m-1) \tau}\right\} \\
Y_{t}=\left\{y_{t}, y_{t+\tau}, y_{t+2 \tau}, \ldots, y_{t+(m-1) \tau}\right\}
\end{gathered}
$$

3) To compute the predictor $X_{t+T}^{*}$, where $T$ is the prediction time, we locate in the learning set the $m+1$ closest neighbours $\left(Y_{k}\right)$ of $X_{t}$. To determine the nearest neighbours, the Euclidean distance is used.

4) The aforementioned neighbours create a cloud of points surrounding the one whose future value we want to predict. We associate a weight, $W_{k}(k=1,2, \ldots, m+1)$, to each neighbour depending on its original distance:

$$
W_{k}=\frac{\left\|X_{t}, Y_{k}\right\|^{-2}}{\sum_{j=1}^{m+1}\left\|X_{t}, Y_{j}\right\|^{-2}}
$$

where $\|\cdot\|$ is the Euclidean distance.

5) The predicted value is obtained by keeping track of where the neighbours move $T$ steps in the future [7]:

$$
X_{t+T}^{*}=\sum_{k=1}^{m+1} W_{k} Y_{k+T}
$$

where $Y_{k+T}$ is the value of the $k^{\text {th }}$ neighbour $T$ steps in the future.

6) Finally, Pearson's linear correlation coefficient between the observed and the predicted time series is calculated. The higher the correlation coefficient, the more accurate the prediction is.

\section{RESULTS}

The previous $N L F$ algorithm was applied to stationary epochs from 148 MEG channels. The attractor was reconstructed using an embedding dimension of $m=9$ and a time delay of $\tau=1$. In our research, the linear correlation coefficient between the observed and the predicted time series was computed from 1 to 10 samples, that is from 5.9 $\mathrm{ms}$ to $59 \mathrm{~ms}$. Afterwards, the prediction curves (correlation coefficient as a function of the prediction time) were plotted. Finally, the slopes of these curves were estimated by a line that fits the data in a least squares sense. 
Fig. 1 illustrates two prediction curves, one obtained from an $\mathrm{AD}$ patient's MEG epoch and other from a control subject's one. This figure shows that the AD patient's curve decreases slowly than the control's one. This behavior is the same for all channels. In Fig. 2, the average slopes of the prediction curves are represented, showing lower values in the control group than in $\mathrm{AD}$ group, indicating that $\mathrm{AD}$ patients' MEGs are more predictable than controls' recordings.

Finally, a receiver operating characteristic (ROC) curve was used to assess the ability of $N L F$ to discriminate AD patients from controls. This method summarizes the performance of a two-class classifier across the range of possible thresholds. Mean values, obtained averaging the results of all channels, were used in this statistical analysis. We achieved and accuracy of $76.7 \%$ ( $80.0 \%$ sensitivity; $73.3 \%$ specificity) at the optimum threshold (-0.044), as Fig. 3 shows.

\section{DISCUSSION AND CONCLUSION}

We analyzed the MEG background activity from 15 patients with probable $\mathrm{AD}$ and 15 age-matched control subjects by means of a $N L F$ measure. Our purpose was to check the hypothesis that MEG background activity was different in $\mathrm{AD}$ patients than in control subjects. $N L F$ has proven to be effective in discriminating $\mathrm{AD}$ patients from controls. Our results revealed that the slope of the prediction curves were lower for the controls than for the patients.

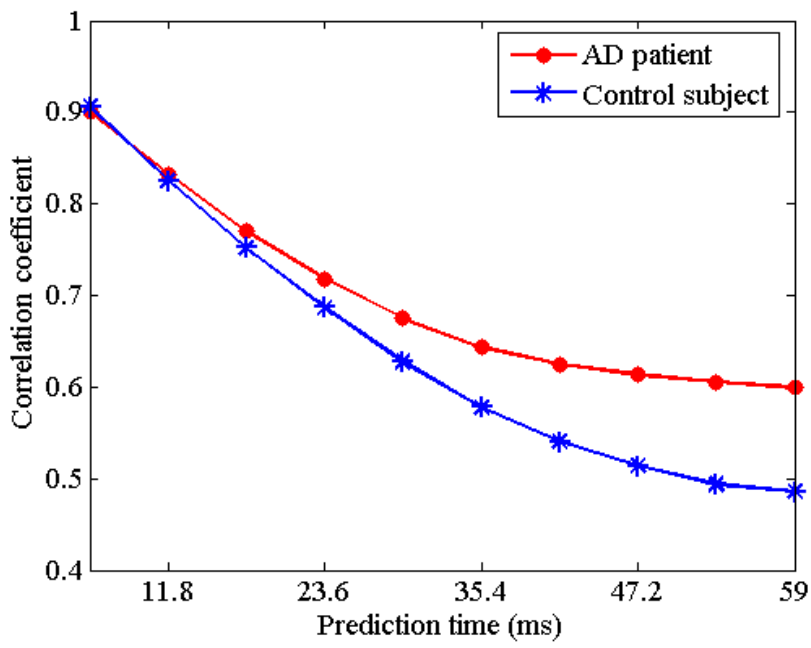

Fig. 1. Prediction curves obtained from an AD patient's MEG epoch and a control subject's one.

Thus, our results suggest that AD patients' MEGs are more predictable than controls subjects' signals. Additionally, an accuracy of $76.7 \%$ was reached at a threshold of -0.044 by means of a ROC curve. Because both groups were carefully matched for age, the significantly reduced MEG predictability may well represent the cognitive dysfunction in $\mathrm{AD}$.

Other non-linear methods have been already used to study the MEG activity in AD. Stam el al. [10] found changes of long and short distances interaction in the frequency bands
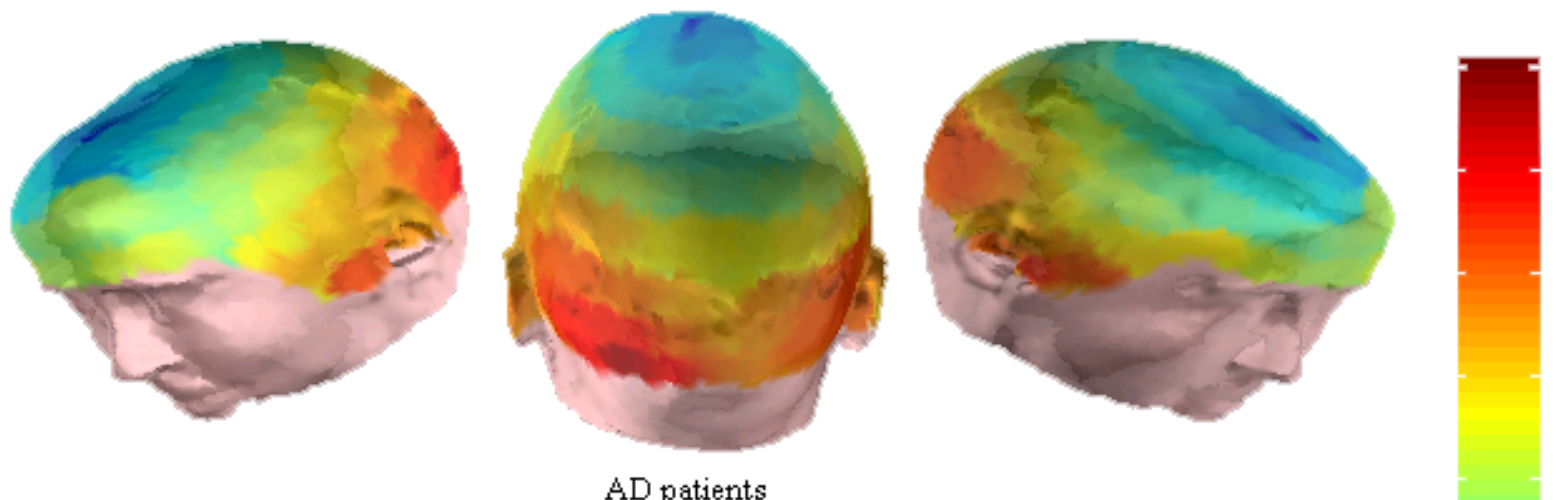

$-0.0319$

AD patients
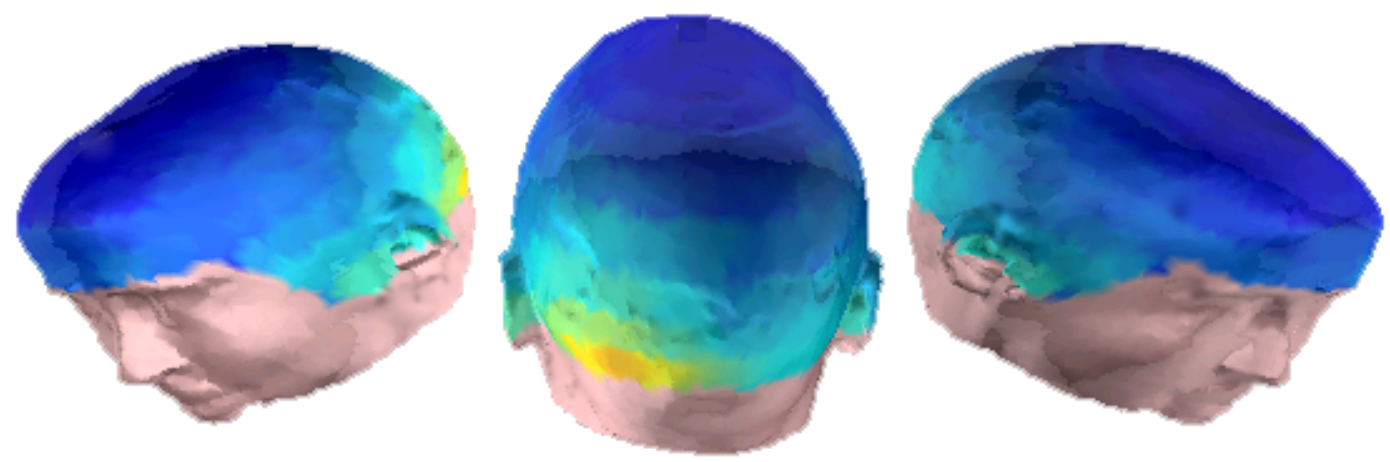

$-0.0428$

$-0.0450$

$-0.0472$

$-0.0494$

Control subjects

Fig. 2. Average slopes of the prediction curves for the $\mathrm{AD}$ group and the control group. 


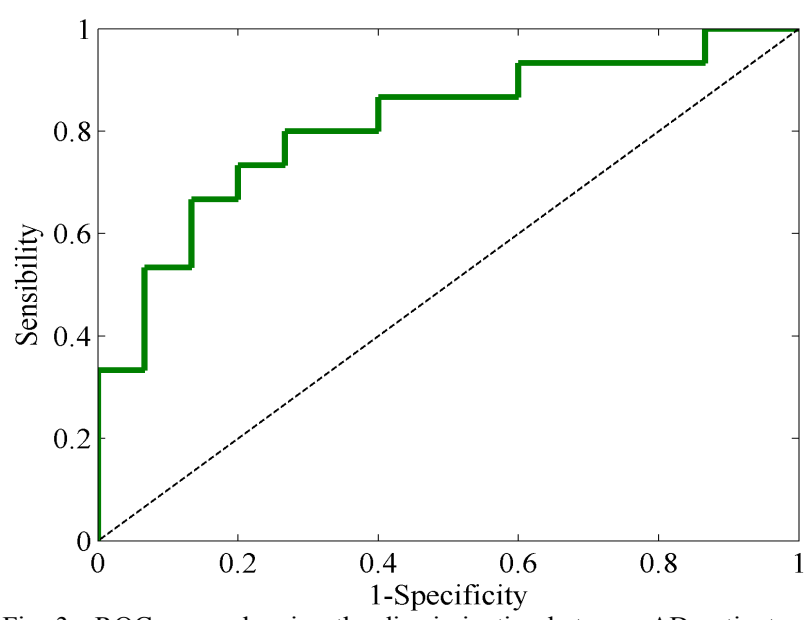

Fig. 3. ROC curve showing the discrimination between $\mathrm{AD}$ patients and control subjects

theta, alpha1, beta and gamma using the synchronization likelihood. These changes may reflect loss of anatomical connections and/or reduced central cholinergic activity and could underlie part of the cognitive impairment [10]. Other MEG studies suggested that brains affected by AD show a more regular physiological behaviour [9, 11]. In [8] and [9], Lempel-Ziv complexity values were significantly lower in the recordings from $\mathrm{AD}$ patients than in those obtained from control subjects. Using correlation dimension, other study revealed a decreased complexity of the MEG background activity in $\mathrm{AD}$ patients in the low frequency bands, and an increased in the high bands [7].

Yunfan et al. [20] suggested that $N L F$ is more efficient and robust than classical measures like correlation dimension or highest Lyapunov exponent for analyzing short and noisy time series. Nevertheless, some limitations of our study merit consideration. Firstly, the sample size is small. Thus, a larger database is needed to confirm our results. Secondly, the detected decrease in predictability is not specific to $\mathrm{AD}$ and it appears in other pathological states [18]. Additionally, our results do not show if $N L F$ can detect a gradation of the disease process. Therefore, future studies are necessary to analyse MEGs from patients with different stages of AD and with Mild Cognitive Impairment.

To sum up, our results suggest that $N L F$ measure is a good method to differentiate between $\mathrm{AD}$ patients and elderly controls. Nevertheless, this is a preliminary study and a larger database is needed to confirm our results.

\section{ACKNOWLEDGMENT}

The authors would like to thank the "Asociación de Familiares de Enfermos de Alzheimer" in Spain for supplying the patients who took part in this study.

\section{REFERENCES}

[1] T. D. Bird, "Alzheimer's disease and other primary dementias," in Harrison's principles of internal medicine, E. Braunwald, A. S. Fauci, D. L. Kasper, S. L. Hauser, D. L. Longo, and J. L. Jameson, Eds. New York: The McGraw-Hill Companies Inc, 2001, pp. 2391-2399.
[2] J. Jeong, "EEG dynamics in patients with Alzheimer's disease," Clin. Neurophysiol., vol. 115, pp. 1490-1505, 2004.

[3] J. L. Cummings, “Alzheimer's disease," N. Engl. J. Med., vol. 351, pp. 56-67, 2004.

[4] C. J. Stam, "Nonlinear dynamical analysis of EEG and MEG: review of an emerging field," Clin. Neurophysiol., vol. 116, pp. 2266-2301, 2005.

[5] R. Hari, "Magnetoencephalography in clinical neurophysiologica assessment of human cortical functions," in Electroencephalography: basic principles, clinical applications, and related fields, 5th ed., E. Niedermeyer, and F. Lopes da Silva, Eds. Philadelphia: Lippincontt Williams \& Wilkins, 2005, pp. 1165-1197.

[6] J. Jeong, S. J. Kim, and S. H. Han, "Non-linear dynamical analysis of the EEG in Alzheimer's disease with optimal embedding dimension," Electroencephalogr. Clin. Neurophysiol., vol. 106, pp. 220-228, 1998.

[7] A. M. van Cappellen van Walsum, Y. A. L. Pijnenburg, H. W Berendse, B. W. van Dijk, D. L. Knol, Ph. Scheltens, and C. J Stam, "A neural complexity measure applied to MEG data in Alzheimer's disease," Clin. Neurophysiol., vol. 114, pp. 1034-1040, 2003.

[8] C. Gómez, R. Hornero, D. Abásolo, A. Fernández, and M. López, "Complexity analysis of the magnetoencephalogram background activity in Alzheimer's disease patients," Med. Eng. Phys., vol. 28, pp. 851-859, 2006.

[9] R. Hornero, J. Escudero, A. Fernández, J. Poza, and C. Gómez, "Spectral and non-linear analyses of MEG background activity in patients with Alzheimer's disease," IEEE Trans. Biomed. Eng., vol. 55 , pp. $1658-1665,2007$.

[10] C. J. Stam, B. F. Jones, I. Manshanden, A. M. van Cappellen van Walsum, T. Montez, J. P. A. Verbunt, J. C. de Munck, B. W. van Dijk, H. W. Berendse, and P. Scheltens, "Magnetoencephalographic evaluation of resting-state functional connectivity in Alzheimer's disease," Neuroimage, vol. 32, pp. 1335-1344, 2006.

[11] C. Gómez, R. Hornero, D. Abásolo, A. Fernández, and J. Escudero, "Analysis of the magnetoencephalogram background activity in Alzheimer's disease patients with auto-mutual information," Comput. Meth. Programs Biomed., vol. 87, pp. 239-247, 2007.

[12] J. Bendat, and A. Piersol, "Random data: analysis and measurement procedures," New York: Willey, 2000.

[13] T. Chau, D. Chau, M. Casas, G. Berall, and D. J. Kenny, "Investigating the stationarity of paediatric aspiration signals," IEEE Trans. Neural Syst. Rehab. Eng., vol. 13, pp. 99-105, 2005.

[14] G. Sugihara, and R. M. May, "Nonlinear forecasting as a way of distinguishing chaos from measurement error in time series," Nature, vol. 344, pp. 734-741, 1990.

[15] L. I. Aftanas, N. V. Lotova, V. I. Koshkarov, S. A. Popov, and V. P. Makhnev, "Nonlinear forecasting measurements of the human EEG during evoked emotions," Brain Topography, vol. 10, pp. 155-162, 1997.

[16] L. Pezard, J. Martinerie, F. Breton, and B. Renault, "Nonlinear forecasting and correlation dimension of brain dynamics: A multichannel study," in Proc. 14th Annu. Int. Conf. IEEE-EMBS, Paris, 1992, pp. 979-981.

[17] J. L. Nandrino, L. Pezard, J. Martinerie., F. L. Massioui, B. Renault, R. Jouvent, J. F. Allilaire, and D. Widlocher, "Decrease of complexity in EEG as symptom of depression," Neuroreport, vol. 5, pp. 528-530, 1994.

[18] C. J. Stam, T. C. A. M. van Woerkom, and R. W. M. Keunen "Nonlinear analysis of the electroencephalogram in Creutzfeldt-Jakob disease," Biol. Cybern., vol. 77, pp. 247-256, 1997.

[19] F. Takens, "Detecting strange attractors in turbulence," Lecture Notes in Mathematics, vol. 898, pp. 366-381, 1981.

[20] G. Yunfan, X. Jianxue, R. Wei, H. Sanjue, and W. Fuzhou, "Determining the degree of chaos from analysis of ISI time series in the nervous system: a comparison between correlation dimension and nonlinear forecasting methods," Biol. Cybern., vol. 78, pp. 159-65, 1998. 